\title{
Tumor infiltrating lymphocytes differ in invasive micropapillary carcinoma and medullary carcinoma of breast
}

\author{
Xiaojing Guo ${ }^{1}$, Yu Fan ${ }^{1}$, Ronggang Lang ${ }^{1}$, Feng Gu ${ }^{1}$, Ling Chen ${ }^{1}$, Lifang $\mathrm{Cui}^{1}$, \\ Gordon A Pringle ${ }^{2}$, Xinmin Zhang ${ }^{2}$ and $\mathrm{Li} \mathrm{Fu}^{1}$ \\ ${ }^{1}$ Department of Breast Cancer Pathology and Research Laboratory, State Key Laboratory of Breast Cancer \\ Research, Cancer Hospital of Tianjin Medical University, Tianjin, China and ${ }^{2}$ Department of Pathology and \\ Laboratory Medicine, Temple University Hospital, Philadelphia, PA, USA
}

\begin{abstract}
Tumor infiltrating lymphocytes have been correlated with a better prognosis for some tumors and medullary carcinoma of breast is a good example. However, in a recent study of invasive micropapillary carcinoma of breast, tumor infiltrating lymphocytes were associated with increased lymph node metastasis and a poorer prognosis. To explore possible mechanisms underlying this difference in immune responsiveness and tumor behavior, 28 cases of invasive micropapillary carcinoma with prominent lymphocyte infiltration were compared with 29 cases of medullary carcinoma. In both tumors, the majority of tumor infiltrating lymphocytes were T lymphocytes $(P<0.01)$ with CD8 + T lymphocytes predominant $(P<0.01)$. Significantly, functional differences in CD8 + cytotoxic T lymphocytes were identified in the two types of tumor. While lymphocytes infiltrated both the stroma and epithelial components of medullary carcinoma, the tumor infiltrating lymphocytes of invasive micropapillary carcinoma were almost exclusively confined to the stroma. Tumor infiltrating lymphocytes of medullary carcinoma showed stronger expression of FasL than those in invasive micropapillary carcinoma $(P<0.01)$ and medullary carcinoma cells exhibited stronger expression of Fas than invasive micropapillary carcinoma cells did $(\boldsymbol{P}<\mathbf{0 . 0 1})$. In the subgroups of tumors with strong $(++I+++)$ Fas expression, double immunohistochemistry revealed that most of the tumor infiltrating lymphocytes in medullary carcinoma, particularly those infiltrating the tumor nests, were CD8 + cytotoxic T lymphocytes, but not so in invasive micropapillary carcinoma. Furthermore, upregulated expression of perforin, granzyme $B$ and FasL by cytotoxic T lymphocytes was greater in medullary carcinoma than invasive micropapillary carcinoma $(P<0.01$, respectively). The results suggest that effective immunity provided by tumor infiltrating lymphocytes varies in different tumors and the relative lack of tumor-killing cytotoxic T lymphocytes in invasive micropapillary carcinoma may explain, in part, the adverse association of tumor infiltrating lymphocytes with the biological behavior of invasive micropapillary carcinoma of breast.
\end{abstract}

Modern Pathology (2008) 21, 1101-1107; doi:10.1038/modpathol.2008.72; published online 9 May 2008

Keywords: tumor infiltrating lymphocytes; invasive micropapillary carcinoma; medullary carcinoma; breast; laser scanning confocal microscope

Local and systemic inflammatory responses play an important role in the progression of a variety of common solid tumors. Tumor infiltrating lymphocytes are believed to represent the local immune response, a key mechanism in controlling tumor growth and metastasis, and an independent prognostic indicator for many tumors. ${ }^{1,2}$ This is well

Correspondence: Dr L Fu, MD, PhD, Department of Breast Cancer Pathology and Research Laboratory, Cancer Hospital of Tianjin Medical University, Huan hu xi Road, Tiyuan Bei, Hexi District, Tianjin 300060, People's Republic of China.

E-mail: fulijyb@hotmail.com

Received 28 January 2008; revised 14 March 2008; accepted 17 March 2008; published online 9 May 2008 demonstrated in medullary carcinoma of breast which is characterized by large sheets of poorly differentiated tumor cells and a prominent lymphoplasmacytic infiltrate, rare lymph node metastasis and a good prognosis. ${ }^{3}$ Invasive micropapillary carcinoma of breast is composed of small clusters of tumor cells lying within clear stromal spaces and exhibits axillary lymph node metastasis with extraordinary frequency. In a previous study of 51 cases of invasive micropapillary carcinoma, 28 cases showed prominent tumor lymphocyte infiltration, ${ }^{4}$ yet this group of patients presented with a significantly higher rate of lymph node metastasis than the group without or with scanty tumor lymphocyte infiltration. To explore the potential mechanisms to 
account for this apparent discordant finding, we compared the immunologic profile and activities of the tumor infiltrating lymphocytes of the 28 cases of invasive micropapillary carcinoma with those in a similar number of cases of medullary carcinoma.

\section{Materials and methods}

\section{Case Selection and Histologic Clarifications}

The 28 cases of invasive micropapillary carcinoma with abundant lymphocytes in the stroma, with or without lymphoid follicle formation, were retrieved from 1056 cases of invasive breast carcinoma diagnosed in a 9-month period during 2004 as described previously. ${ }^{4}$ A total of 29 cases of medullary carcinoma were diagnosed between 2003 and 2006 in the Department of Breast Cancer Pathology and Research Laboratory, Tianjin Medical University Cancer Hospital, Tianjin, China and were retrieved for this study. The criteria for diagnosis of medullary carcinoma were those described in the 2003 World Health Organization histologic classification of tumors of the breast; ${ }^{3}$ namely, syncytial growth pattern $(>75 \%)$, absence of glandular structures, diffuse infiltration of lymphocytes, nuclear pleomorphism and complete histological circumscription. H\&E-stained slides from each case were reviewed independently by two pathologists to confirm the diagnosis and to evaluate the distribution of tumor infiltrating lymphocytes. All cases of medullary carcinoma were females 32-69 years of age (mean age 51 years). The 28 female patients with invasive micropapillary carcinoma were 40-66 years of age (mean age 53 years).

\section{Immunohistochemical Studies}

Immunoperoxidase analysis of sections of formalinfixed tissue from all cases was performed with an avidin-biotin system using a standard protocol. Briefly, $4 \mu \mathrm{m}$ tissue sections on coated slides were heated for antigen retrieval, pretreated with a $3 \%$ solution of hydrogen peroxide for 5-10 min, rinsed and incubated with $5-10 \%$ normal goat serum as a blocking agent. The sections were then incubated sequentially with primary monoclonal antibody (mAb; Zymed Laboratories, CD3 in 1:100 dilution, CD4 in 1:20 dilution, CD8 in 1:75 dilution, CD20 in 1:100 dilution and CD56 in 1:50 dilution), biotinylated secondary antibody and avidin peroxidase conjugate. All steps were preceded by rinsing of sections with $\mathrm{PBS}(\mathrm{pH}$ 7.6). The chromogen was DAB. The immunoreaction for CD3, CD4, CD8, CD20 and CD56 in lymphocytes and Fas (Zymed Laboratories, 1:75) or FasL (Zymed Laboratories, 1:75) in lymphocytes and tumor cells was evaluated independently by two pathologists and graded as follows: $(-)=$ no positive cells, $(+)=1-25 \%$ of the cells stained, $(++)=26-50 \%$ of the cells stained and $(+++)=51-100 \%$ of the cells stained.
In the cases where carcinoma cells expressed Fas strongly $(++/+++)$, double immunoperoxidase studies were carried out to detect expression of CD4/ Fas and CD8/Fas using the histostain-DS KIT (Zymed Cat. No.95-9999). The manufacturer's protocol was followed. Briefly, $4 \mu \mathrm{m}$ tissue sections on coated slides were pretreated with a $3 \%$ solution of hydrogen peroxide for $10 \mathrm{~min}$, rinsed and incubated with $5-10 \%$ normal goat serum as a blocking agent. Following incubation with the first primary $\mathrm{mAb}$ against CD4 (Zymed Laboratories, 1:20) or CD8 (Zymed Laboratories, 1:75) for $60 \mathrm{~min}$ at $37^{\circ} \mathrm{C}$, the sections were then incubated sequentially with biotinylated second antibody and avidin conjugated with alkaline phosphatase. After substrate-chromogen mixture and double staining enhancer were applied to the sections, they were incubated with the second primary mAb against Fas (Zymed Laboratories, 1:75) for $60 \mathrm{~min}$ at $37^{\circ} \mathrm{C}$ and then followed by biotinylated second antibody and avidin peroxidase conjugate and substrate-chromogen mixture was used. All steps were preceded by rinsing of sections with $\mathrm{PBS}(\mathrm{pH}$ 7.5) containing $0.05 \%$ Tween 20 . The lymphocytes positive for CD4 or CD8 were stained dark purple and the tumor cells and lymphocytes positive for Fas were stained an intense red. The positivity of lymphocytes for CD4 or CD8 was graded as follows: $(-)=$ no positive cells, $(+)=1-25 \%$ of the cells stained and $(++)$ $=26-100 \%$ of the cells stained.

To investigate the activities of the CD8 + cytotoxic $\mathrm{T}$ lymphocytes in the tumors, the coexpression of CD8/perforin, CD8/granzyme B, CD8/Fas and CD8/ FasL was evaluated using double immunofluorescence staining with a laser scanning confocal microscope. $4 \mu \mathrm{m}$ tissue-fixed sections on coated slides were heated for antigen retrieval, pretreated with a $3 \%$ solution of hydrogen peroxide for 5-10 min, rinsed and incubated with 5-10\% normal goat serum as a blocking agent. After incubation with rabbit primary mAb against CD8 (Zymed Laboratories, 1:75) for $60 \mathrm{~min}$ at $37^{\circ} \mathrm{C}$, the sections were incubated with FITC-conjugated goat antirabbit IgG (Zymed Laboratories, 1:100) for $30 \mathrm{~min}$ at $37^{\circ} \mathrm{C}$. They were further incubated with mouse primary $\mathrm{mAb}$ against perforin (Zymed Laboratories, 1:20), granzyme B (Zymed Laboratories, 1:100), Fas (Zymed Laboratories, 1:75) or FasL (Zymed Laboratories, 1:75) for $60 \mathrm{~min}$ at $37^{\circ} \mathrm{C}$ and then incubated with TRITC-conjugated goat anti-mouse IgG (Zymed Laboratories, 1:100) for $30 \mathrm{~min}$ at $37^{\circ} \mathrm{C}$. All steps were preceded by rinsing of sections with PBS ( $\mathrm{pH}$ 7.6). The immunofluorescence images were visualized using a laser scanning confocal microscope (TCS-NT; Leica Microsystems Inc., Exton, PA). Separate images for FITC and TRITC staining were captured digitally from the double-stained specimens using an AxioCam digital camera (Carl Zeiss, Göttingen, Germany) and AxioVision 2.05 multichannel image processor (Carl Zeiss Vision GmbH, Hallbergmoos, Germany). The red TRITC and green 

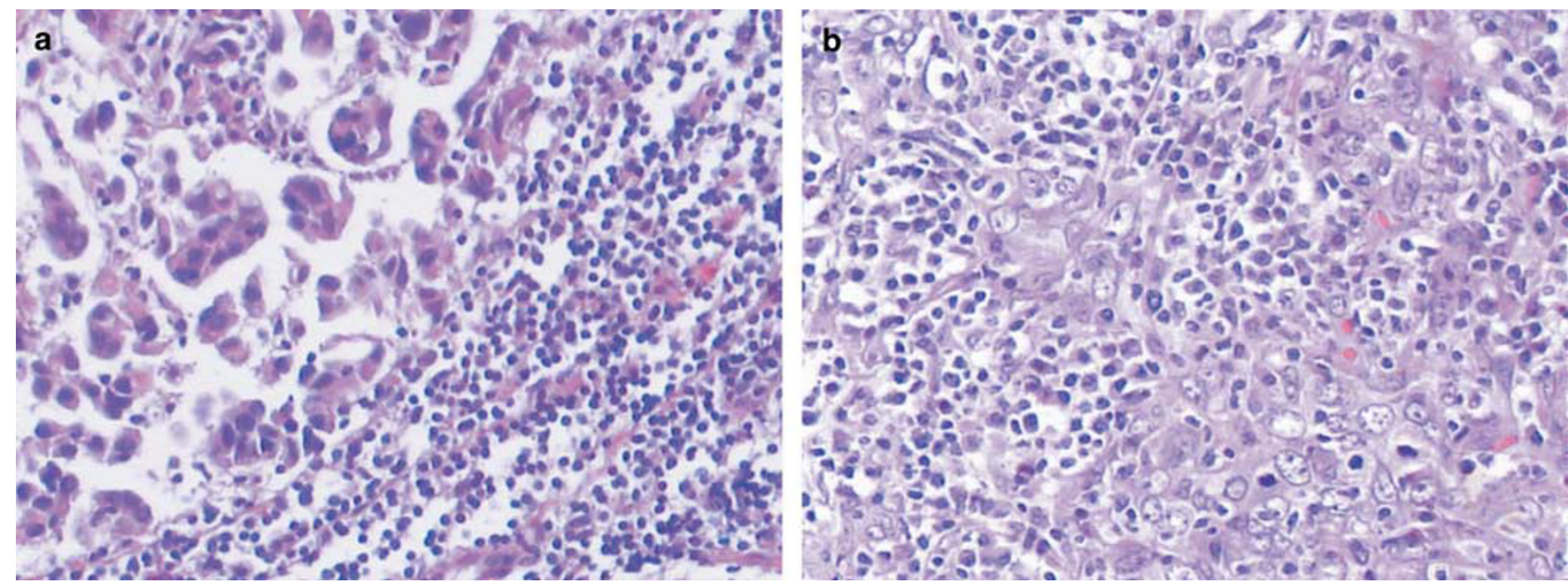

Figure 1 Hematoxylin and eosin sections showing tumor infiltrating lymphocytes' infiltration in the stroma of invasive micropapillary carcinoma (a, $\times 200$ magnification) and in both the tumor nests (intraepithelial) and the stroma of medullary carcinoma (b, $\times 200$ magnification).

FITC-stained images were merged digitally to create the yellow stain indicative of active CD8 + cytotoxic $\mathrm{T}$ lymphocytes expressing both antigens. Laser lines and emission filters were optimized with software (PowerScan Leica). Confocal images were analyzed with the confocal software (Leica).

Immunohistochemistry for estrogen/progesterone receptors and HER-2/neu was also performed using standard procedures.

\section{Statistics}

Statistical analyses were performed using Wilcoxon signed-ranks test and Mann-Whitney test.

\section{Results}

\section{Histologic Grade and Lymph Node Metastasis}

Lymph node metastasis was present in 27 of the 28 $(96 \%)$ cases of invasive micropapillary carcinoma with prominent tumor infiltrating lymphocytes. Lymph node metastasis was found in only 3 of 29 $(10 \%)$ cases of medullary carcinoma $(P<0.01)$. In 24 of the 28 cases of invasive micropapillary carcinoma and in all cases of medullary carcinoma, the tumors were of high or intermediate nuclear grade and the difference between the two groups in terms of histologic grade was not significant $(P=0.052)$ (data not shown).

\section{Hormone Receptors and HER-2/neu Status}

A total of $19(68 \%), 14(50 \%)$ and 11 (39\%) of the 28 cases of invasive micropapillary carcinoma were positive for estrogen receptor, progesterone receptor and HER-2/neu respectively, in contrasting to the medullary carcinomas, which were positive for estrogen and progesterone receptors in 3 of 29 cases
$(10 \%)$ and none of them was positive for HER-2/neu. The findings were similar to those reported in literature..$^{5,6}$

\section{Distribution of Tumor Infiltrating Lymphocytes}

Tumor infiltrating lymphocytes were confined to the stroma of invasive micropapillary carcinoma almost exclusively (Figure 1a), whereas tumor infiltrating lymphocytes in medullary carcinoma were prominent in both the tumor nests (intraepithelial) and the stroma (Figure 1b).

\section{Immunohistochemical Analysis}

In both types of breast carcinoma, $\mathrm{T}$ lymphocytes were more numerous than $B$ lymphocytes as the predominant tumor infiltrating lymphocytes $(P<0.01$, respectively; Table 1$)$ and CD8 + cells were the major component of the T-lymphocyte population $(P<0.01$, respectively). The results also indicated that comparable numbers of CD3 + , CD4 ,$+ \mathrm{CD} 8+$ and CD20 + cells were present in both types of tumor (Table 1). Noticeable numbers of NK cells were identified in only one case of each tumor.

More medullary carcinoma cells expressed Fas than did the carcinoma cells of invasive micropapillary carcinoma $(P<0.01$; Figure 2a and b) and more tumor infiltrating lymphocytes in medullary carcinoma were stained for FasL than tumor infiltrating lymphocytes in invasive micropapillary carcinoma $(P<0.01$; Figures $2 \mathrm{c}$ and $\mathrm{d})$. However, the expression of Fas by tumor infiltrating lymphocytes and FasL by carcinoma cells were not significantly different between the two tumors $(P=0.157$ and $P=0.150$, respectively; Table 2$)$.

Strong expression $(++$ and +++$)$ of Fas by carcinoma cells occurred in 25 cases of medullary carcinoma and seven cases of invasive micropapillary carcinoma. Double immunoperoxidase analysis 
Table 1 CD3, CD4, CD8 and CD20 immunohistochemistry

\begin{tabular}{|c|c|c|c|c|c|c|c|c|c|c|c|c|c|c|c|c|c|}
\hline & \multirow[t]{2}{*}{$\mathrm{N}$} & \multicolumn{4}{|c|}{$C D 3$} & \multicolumn{4}{|c|}{$C D 20$} & \multicolumn{4}{|c|}{$C D 4$} & \multicolumn{4}{|c|}{$C D 8$} \\
\hline & & - & + & ++ & +++ & - & + & ++ & +++ & - & + & ++ & +++ & - & + & ++ & +++ \\
\hline Invasive micropapillary carcinoma & 28 & 0 & 1 & 12 & $15^{*}$ & 1 & 21 & 6 & $0^{*}$ & 0 & 11 & 15 & $2^{\dagger}$ & 0 & 3 & 12 & $13^{\dagger}$ \\
\hline Medullary carcinoma & 29 & 0 & 1 & 10 & $18^{\ddagger}$ & 1 & 20 & 8 & $0^{\ddagger}$ & 0 & 15 & 14 & $0^{\S}$ & 0 & 3 & 11 & $15^{\S}$ \\
\hline
\end{tabular}

*: $Z=-4.824, P<0.01 ;{ }^{\dagger}: Z=-4.359, P<0.01$.

: $Z=-4.893, P<0.01 ; \stackrel{s}{ }: Z=-5.014, P<0.01$ (Wilcoxon signed-ranks test).
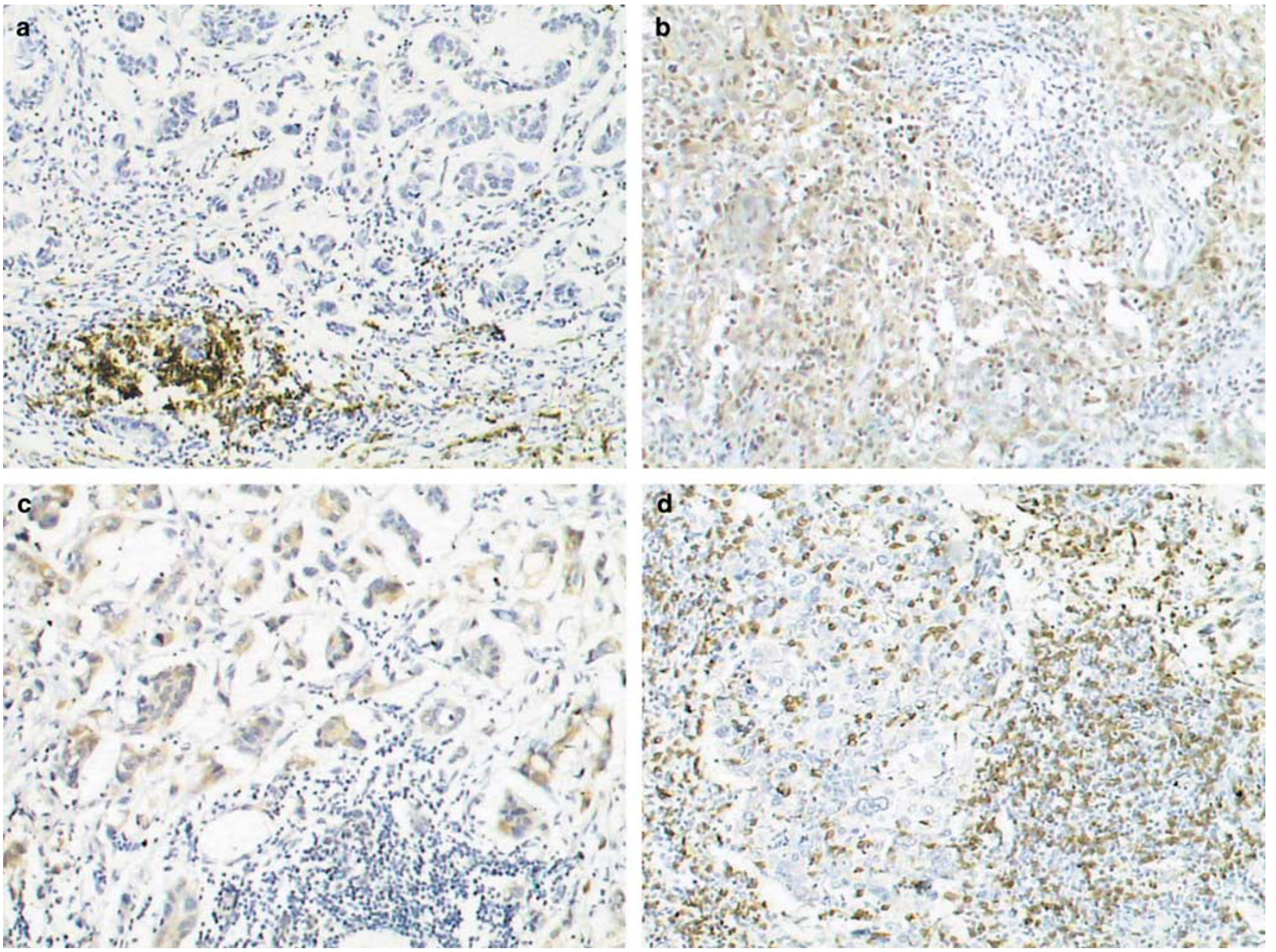

Figure 2 Immunohistochemistry for Fas showing fewer carcinoma cells labeled in invasive micropapillary carcinoma $(\mathbf{a}, \times 100$ magnification) than those in medullary carcinoma $(\mathbf{b}, \times 100$ magnification) and fewer lymphocytes labeled by FasL in invasive micropapillary carcinoma $(\mathbf{c}, \times 100$ magnification) than those in medullary carcinoma $(\mathbf{d}, \times 100$ magnification).

of these subgroups of both tumors revealed that majority of the tumor infiltrating lymphocytes in all 25 cases of medullary carcinoma, particularly those infiltrating between the carcinoma cells, were CD8 + cells $(P<0.01)$. The lymphocytes in the stroma of invasive micropapillary carcinoma were more of an even mixture of CD4 + and CD8 + cells $(P=0.083)$ (data not shown).

Stronger expressions of perforin, granzyme B and FasL were noted in CD8 + cytotoxic T lymphocytes of medullary carcinoma than those in invasive micropapillary carcinoma by double immunofluor- escence analysis $(P<0.01$, respectively; Table 3 ; Figure 3a-d). Expression of Fas by the CD8 + cells was not significantly different between the two tumor groups $(P=0.078)$, which agrees with the data in Table 2.

\section{Discussion}

Tumor infiltrating lymphocytes form an important aspect of the host defense against the expansion of solid neoplasms. It is the general belief that recruitment 
and accumulation of high concentrations of lymphocytes in the tumor tissue represent an important local barrier to neoplastic dissemination. Tumor infiltrating lymphocytes are a key feature of medullary carcinoma of breast, which despite of the characteristic cytological anaplasia, has a more favorable prognosis than invasive micropapillary carcinoma. It has been proposed that the improved clinical outcome is due at least in part to the presence of prominent lymphocyte infiltrates in the tumor. ${ }^{7-11}$ In contrast, invasive micropapillary carcinoma has a high incidence of axillary lymph node metastasis and poor prognosis and the mechanism is still poorly understood. In our previous study, ${ }^{4}$ we found that invasive micropapillary carcinoma rich in tumor infiltrating lymphocytes was associated with an increased incidence of lymph node metastasis and an increased number of positive lymph nodes per case. A similar adverse effect of tumor infiltrating lymphocytes was also reported in primary cutaneous melanoma. ${ }^{12}$ Why tumors rich in tumor infiltrating lymphocytes have significant differences in behavior and clinical courses is unknown, although several mechanisms have been suggested in the melanoma immunity. ${ }^{13}$ We hypothesized that the functional status of tumor infiltrating lymphocytes are different in different tumors, which might be important in the tumor immunity.

$\mathrm{T}$ lymphocytes have been reported to be the predominant tumor infiltrating lymphocytes in

Table 2 Fas and FasL immunohistochemistry

\begin{tabular}{|c|c|c|c|c|c|c|c|c|c|}
\hline & \multirow[t]{2}{*}{$\mathrm{N}$} & \multicolumn{4}{|c|}{ Fas } & \multicolumn{4}{|c|}{ Fas $L$} \\
\hline & & - & + & ++ & +++ & - & + & ++ & +++ \\
\hline \multicolumn{10}{|c|}{ Tumor cells } \\
\hline $\begin{array}{l}\text { Invasive micropapillary } \\
\text { carcinoma }\end{array}$ & 28 & 2 & 19 & 5 & $2^{*}$ & 4 & 14 & 8 & $2^{\dagger}$ \\
\hline Medullary carcinoma & 29 & 0 & 4 & 15 & $10^{*}$ & 6 & 18 & 4 & $1^{\dagger}$ \\
\hline \multicolumn{10}{|c|}{ Infiltrating lymphocytes } \\
\hline $\begin{array}{l}\text { Invasive micropapillary } \\
\text { carcinoma }\end{array}$ & 28 & 4 & 13 & 9 & $2^{\ddagger}$ & 2 & 18 & 6 & $2^{\S}$ \\
\hline Medullary carcinoma & 29 & 6 & 17 & 5 & $1^{\ddagger}$ & 0 & 4 & 16 & $9^{\S}$ \\
\hline
\end{tabular}

*: $Z=-4.462, P<0.01 ;{ }^{\dagger}: Z=-1.438, P=0.150$.

*: $Z=-1.416, P=0.157 ;$ \$ $Z=-4.242, P<0.01$ (Mann-Whitney test). breast carcinomas and the findings of our study (Table 1) are consistent with the results reported previously. ${ }^{14-17}$ NK cells were only noted in rare cases. It has also been reported that active CD8 + cytotoxic $\mathrm{T}$ lymphocytes are the principal cells attacking breast carcinoma cells. ${ }^{18-20}$ In medullary carcinoma of breast, our results strongly suggest that there is an active immune response against the tumor. Tumor infiltrating lymphocytes were typically present in both the stroma and within the epithelial nests of tumor cells. Direct contact of cytotoxic $\mathrm{T}$ lymphocytes with tumor cells is required for active antitumor immune reactions, notably the process of tumor cell apoptosis. FAS receptor and Fas ligand are complementary members of an immune apoptotic pathway with activation of FasL triggering cytotoxic mechanisms leading to the death of Fas-expressing target cells. Both tumor cells and tumor infiltrating lymphocytes may express Fas and FasL in various proportions and their interplay may affect tumor behavior. In this study, medullary carcinoma cells demonstrated upregulated expression of Fas and the tumor infiltrating lymphocytes in medullary carcinoma demonstrated increased expression of FasL (Table 2). Furthermore, cases of medullary carcinoma expressing high levels of Fas were infiltrated by large number of CD8 + lymphocytes. The fact that these CD8 + cells were active cytotoxic T lymphocytes is supported by the double immunoflurorescence studies indicating that these cells expressed high levels of perforin, granzyme $B$ and FasL. Therefore, all the tools for a strong immune reaction against medullary carcinoma tumor cells are identified and this immunity has been postulated to ensure a more favorable prognosis for this tumor.

These findings for medullary carcinoma contrast sharply with those of invasive micropapillary carcinoma. In invasive micropapillary carcinoma, the tumor infiltrating lymphocytes exhibit a different pattern of infiltration in which they are confined almost exclusively to the connective tissue stroma. The lack of infiltration of tumor infiltrating lymphocytes among the carcinoma cells would physically limit the direct contact needed for cytotoxic T-cell killing. The expression of Fas by invasive micropapillary carcinoma tumor cells and FasL by the tumor infiltrating lymphocytes in invasive micropapillary carcinoma was also significantly less

Table 3 Double immunofluorescence analysis

\begin{tabular}{lccccc}
\hline & $\mathrm{N}$ & $\begin{array}{c}\text { Percentage of } \\
\text { perforin }{ }^{+} C D 8^{+}\end{array}$ & $\begin{array}{c}\text { Percentage of } \\
\text { granzymeB }{ }^{+} C D 8^{+}\end{array}$ & $\begin{array}{c}\text { Percentage of } \\
\text { FasL }^{+} C D 8^{+}\end{array}$ & $\begin{array}{c}\text { Percentage of } \\
\text { Fas }^{+} C D 8^{+}\end{array}$ \\
\hline $\begin{array}{l}\text { Invasive micropapillary } \\
\text { carcinoma }\end{array}$ & 28 & $0.320^{*}$ & $0.355^{\dagger}$ & $0.370^{\ddagger}$ & $0.505^{\S}$ \\
\begin{tabular}{l} 
Medullary carcinoma \\
\hline
\end{tabular} & 29 & $0.690^{*}$ & $0.740^{\dagger}$ & $0.670^{\ddagger}$ & $0.470^{\S}$ \\
\hline
\end{tabular}

${ }^{*}: Z=-6.466, P<0.01{ }^{\dagger}: Z=-6.483, P<0.01$.

‡: $Z=-6.118, P<0.01$; § : $Z=-1.831, P=0.078$ (Mann-Whitney test). 

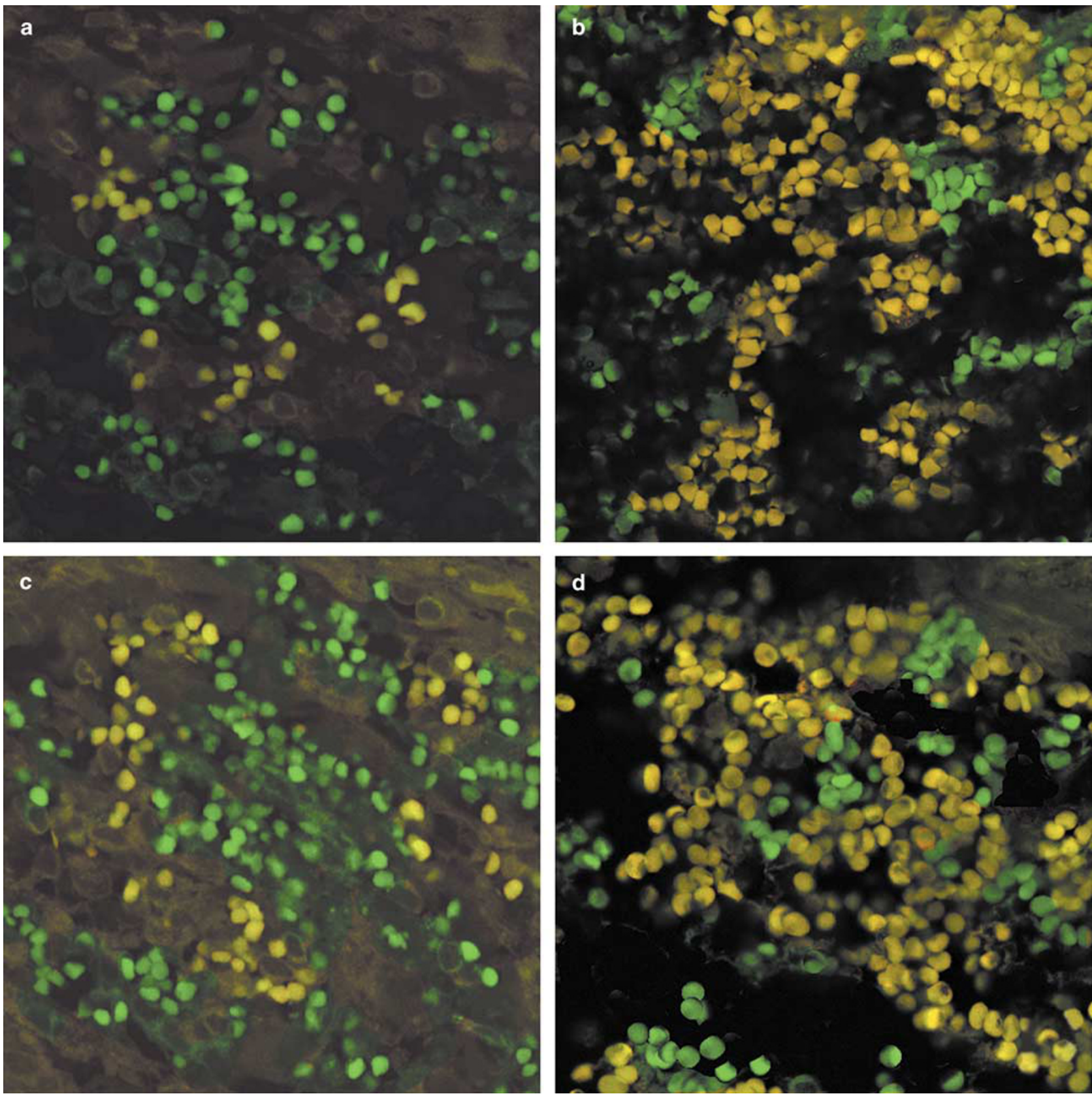

Figure 3 Double immunofluorescence analysis showing stronger expression of perforin in CD8 + lymphocytes of medullary carcinoma (b) than those in invasive micropapillary carcinoma (a) and stronger expression of granzyme B in CD8 + lymphocytes of medullary carcinoma (d) than those in invasive micropapillary carcinoma (c). The green color indicates CD8-positive lymphocytes and the yellow color represents the perforin ${ }^{+} \mathrm{CD}^{+}$or granzyme $\mathrm{B}^{+} \mathrm{CD}^{+}$lymphocytes.

than that demonstrated by medullary carcinoma (Table 2). Furthermore, the tumor infiltrating lymphocytes of invasive micropapillary carcinoma with strong Fas expression by carcinoma cells were not predominantly CD8 + cells and the CD8 + cytotoxic T lymphocytes demonstrated significantly less activation (Table 3). Thus, while both types of tumor had comparable influx of CD4 + and CD8 + lymphocytes (Table 1), it would appear that the presence of tumor infiltrating lymphocytes per se does not guarantee tumor containment and a better prognosis.
The results of this study suggest that there is a relative lack of development of an efficient immune reaction by cytotoxic $\mathrm{T}$ lymphocytes against invasive micropapillary carcinoma cells. Indeed, only 28 of 51 cases $(55 \%)$ of invasive micropapillary carcinoma demonstrated a prominent lymphocytic infiltrate. Mechanisms include a lack of tumorassociated or tumor-specific antigens expressed by invasive micropapillary carcinoma, active downregulation of Fas, defects in the mechanisms of gaining close access to epithelial cells, as well as the findings of recent studies that indicate that tumors 
may be able to regulate the infiltration and activity of host cells in ways that promote tumor survival and progression. ${ }^{21-24}$ Of further interest is the finding that cases of invasive micropapillary carcinoma with a heavy lymphocytic infiltrate are more likely to metastasize. This finding suggests that there may not be only a relative lack of tumor immunity, but that the immune components present facilitate tumor spread. Key to the understanding of this possibility is an elucidation of the functional status and role of the CD8 + lymphocytes that are present and whether local production of cytokines, proteases and angiogenic factors by inflammatory cells outweighs the immune response and promotes tumor growth, angiogenesis and metastasis. Further studies are needed to elucidate the potential dual role of tumor infiltrating lymphocytes in host defense and tumor progression.

In summary, this study revealed that the functional and activation status of tumor infiltrating lymphocytes are different in medullary carcinoma and invasive micropapillary carcinoma, and it is suggested that these differences are associated with the relative progression of each of these tumors. Functional evaluation of tumor infiltrating lymphocytes might be an important asset in predicting tumor behavior and prognosis.

\section{Acknowledgement}

Financial support: Supported by funding from National Natural Science Foundation of China (30600225 and 30470667).

\section{References}

1 Tsuta K, Ishii G, Kim E, et al. Primary lung adenocarcinoma with massive lymphocyte infiltration. Am J Clin Pathol 2005;123:547-552.

2 Canna K, McArdle PA, McMillan DC, et al. The relationship between tumour T-lymphocyte infiltration, the systemic inflammatory response and survival in patients undergoing curative resection for colorectal cancer. Br J Cancer 2005;92:651-654.

3 Tavassoli FA, Devilee P. WHO Classification of Tumours. Pathology \& Genetics, Tumours of the Breast and Female Genital Organs. IARC Press: Lyon, 2003, 28-29.

4 Guo X, Chen L, Lang R, et al. Invasive micropapillary carcinoma of the breast Association of pathologic features with lymph node metastasis. Am J Clin Pathol 2006;126:740-746.

5 Zekioglu O, Erhan Y, Ciris M, et al. Invasive micropapillary carcinoma of the breast: high incidence of lymph node metastasis with extranodal extension and its immunohistochemical profile compared with invasive ductal carcinoma. Histopathology 2004;44: 18-23.

6 Vincent-Salomon A, Gruel N, Lucchesi C, et al. Identification of typical medullary breast carcinoma as a genomic sub-group of basal-like carcinomas, a heterogeneous new molecular entity. Breast Cancer Res 2007;9:R24.

7 Rapin V, Contesso G, Mouriesse H, et al. Medullary breast carcinoma. A reevaluation of 95 cases of breast cancer with inflammatory stroma. Cancer 1998;61: 2503-2510.

8 Wargotz ES, Silverberg SG. Medullary carcinoma of the breast: a clinicopathologic study with appraisal of current diagnostic criteria. Hum Pathol 1988;19:1340-1346.

9 Ridolfi RL, Rosen PP, Port A, et al. Medullary carcinoma of the breast: a clinicopathologic study with 10-year follow-up. Cancer 1997;40:1365-1385.

10 Coronella JA, Spier C, Welch M, et al. Antigen-driven oligoclonal expansion of tumor-infiltrating $B$ cells in infiltrating ductal carcinoma of the breast. J Immunol 2002;169:1829-1836.

11 Nzula S, Going JJ, Stott DI. Antigen driven clonal proliferation, somatic hypermutation, and selection of B lymphocytes infiltrating human ductal breast carcinomas. Cancer Res 2003;63:3275-3280.

12 Clemente CG, Mihm Jr MC, Bufalino R, et al. Prognostic value of tumor infiltrating lymphocytes in the vertical growth phase of primary cutaneous melanoma. Cancer 1996;77:1303-1310.

13 Gajewski TF. Identifying and overcoming immune resistance mechanisms in the melanoma tumor microenvironment. Clin Cancer Res 2006;12:2326s-2330s.

14 Inokuma M, dela Rosa C, Schmitt C, et al. Functional T cell responses to tumor antigens in breast cancer patients have a distinct phenotype and cytokine signature. J Immunol 2007;179:2627-2633.

15 Leong PP, Mohammad R, Ibrahim N, et al. Phenotyping of lymphocytes expressing regulatory and effector markers in infiltrating ductal carcinoma of the breast. Immunol Lett 2006;102:229-236.

16 Ben-Hur H, Cohen O, Schneider D, et al. The role of lymphocytes and macrophages in human breast tumorigenesis: an immunohistochemical and morphometric study. Anticancer Res 2002;22:1231-1238.

17 Wong PY, Staren ED, Tereshkova N, et al. Functional analysis of tumor-infiltrating leukocytes in breast cancer patients. J Surg Res 1998;76:95-103.

18 Yakirevich E, Izhak OB, Rennert G, et al. Cytotoxic phenotype of tumor infiltrating lymphocytes in medullary carcinoma of the breast. Mod Pathol 1999;12:1050-1056.

19 Tamiolakis D, Simopoulos C, Cheva A, et al. Immunophenotypic profile of tumor infiltrating lymphocytes in medullary carcinoma of the breast. Eur J Gynaecol Oncol 2002;23:433-436.

20 Kuroda H, Tamaru J, Sakamoto G, et al. Immunophenotype of lymphocytic infiltration in medullary carcinoma of the breast. Virchows Arch 2005;446:10-14.

21 Schaider H, Oka M, Bogenrieder T, et al. Differential response of primary and metastatic melanomas to neutrophils attracted by IL-8. Int J Cancer 2003;103: 335-343.

22 Shamamian P, Schwartz JD, Pocock BJ, et al. Activation of progelatinase A (MMP-2) by neutrophil elastase, cathepsin G, and proteinase-3: a role for inflammatory cells in tumor invasion and angiogenesis. J Cell Physiol 2001;189:197-206.

23 Scapini P, Nesi L, Morini M, et al. Generation of biologically active angiostatin kringle 1-3 by activated human neutrophils. J Immunol 2002;168:5798-5804.

24 Lin EY, Pollard JW. Role of infiltrated leucocytes in tumour growth and spread. Br J Cancer 2004;90:2053-2058. 